\title{
Configuración de la Agenda en la Unión Europea*
}

\author{
Agenda-Setting in the European Union
}

\author{
Petya Alexandrova \\ Leibniz University Hannover \\ Marcello Carammia \\ University of Malta
}

doi: $10.18543 /$ ced-55-2016pp33-59

\begin{abstract}
Sumario: I. Introducción.-II. Ámbito de investigación. 1. La aproximación teórica-racional. 2. La aproximación teórica-racional limitada.III. Enfoque empírico de la investigación sobre el establecimiento de agenda en la UE. 1. Actores y escenarios. 2. Procesos, asuntos y «areas de interés».-IV. Conceptos clave y argumentos.-V. Herramientas de investigación.-VI. Conclusión.
\end{abstract}

Resumen: El establecimiento de la agenda es un proceso selectivo en el que los asuntos ganan la atención de los formuladores de políticas (policy-makers) y se les atribuye prioridad. Dentro del contexto de la UE se han aplicado dos grandes marcos teóricos para estudiar este proceso - uno basado en la elección racional y otro en la racionalidad limitada - . La investigación en el primer ámbito se ha centrado en los actores y el poder de configuración de la agenda (de carácter condicional), mientras que el segundo ha hecho más hincapié en las restricciones cognitivas, institucionales y externas al procesamiento de la información. Dentro de este último las teorías importantes han sido: 1) la teoría de corrientes múltiples; y 2) la teoría del equilibrio interrumpido. Respecto al enfoque empírico del establecimiento de agenda de la UE, podemos distinguir dos ámbitos. Los análisis de actores o escenarios intentan clarificar las relaciones de poder y la composición de agenda dentro de las instituciones. Los análisis de asuntos específicos (o las áreas de las políticas públicas) rastrean el camino que sigue un tema determinado en la agenda. Respecto a las principales características del sistema político en relación al establecimiento de agenda, algunas características destacadas son la coexistencia de rutas formales e informales de acceso, la competición entre niveles de jurisdicción, y diferentes rutas de configuración de agenda en relación a los principales escenarios de la acción política. De esta manera, el campo de configuración de agenda es rico tanto en marcos de análisis como en aproximaciones metodológicas.

Palabras clave: Unión Europea, configuración de agenda, política pública.

* Recibido el 19 de mayo de 2016, aceptado el 21 de junio de 2016. 
Abstract: Agenda setting is a selective process in which issues gain policy makers' attention and become prioritised. Within the EU context two major theoretical frameworks have been applied to study this process - one based on rational choice assumptions and one on bounded rationality. Research within the first has concentrated on actors and (conditional) agenda setting power, while the second has emphasised cognitive, institutional and external constrains to information processing. Within the latter particularly prominent theories have been multiple streams and punctuated equilibrium. In regard to the empirical focus of EU agenda setting, two main domains can be distinguished. Analyses of actors or venues aim to disentangle power relationships and agenda composition within institutions. Analyses of specific issues (or policy areas) trace the evolutionary path of the issue on the agenda. With respect to the main characteristics of the political system in relation to agenda setting, distinct features are the coexistence of formal and informal routes of access, the competition between levels of jurisdiction, and different agenda-setting routes in relation to the main policy venues involved. The field of EU agenda setting is therefore rich in terms of both analytical frameworks and methodological approaches.

Keywords: European Union, agenda setting, public policy.

\section{Introducción}

¿Por qué la Unión Europea (UE) se centra en determinados problemas políticos mientras ignora otros? ¿Qué significado tiene este proceso de selección y priorización para el sistema político de la UE? El conjunto de problemas potenciales que los sistemas políticos podrían tener en un momento determinado es extremadamente amplio, pero el recurso esencial para detectar dichos problemas, la atención, es limitado. La cantidad de atención prestada no es necesariamente proporcional a la severidad de los problemas detectados. Al contrario: los actores políticos tienden a ignorar habitualmente problemas durante el mayor tiempo posible, cosa que se traduce en una especie de sub-reacción. Cuando se alcanza un determinado umbral, esta práctica se sigue con una respuesta exagerada para compensar la ignorancia anterior. El acceso de los problemas a la agenda política y su priorización es fundamentalmente un proceso político, que implican elecciones explícitas pero limitadas. Entonces, el estudio del establecimiento de la agenda se ocupa tanto de las decisiones en políticas públicas como de los procesos políticos tras estas decisiones, que nos ofrecen una mayor introspección del funcionamiento de todo el sistema político.

La investigación en el establecimiento de agenda tiene su origen en los EUA en los años 60, en base a la tradición de estudios de poder polí- 
tico $^{1}$, y se ha expandido desde entonces ${ }^{2}$. Desde los años 90 , este campo de estudio ha dado un paso adelante con el desarrollo de aproximaciones analíticas innovadoras y un cambio en el ámbito de interés más allá de los EUA, también en perspectiva comparada ${ }^{3}$.

En el contexto de la UE, investigaciones académicas sobre el establecimiento de agenda tomó una senda distinta. Hasta los años 90, el discurso dominante conceptualizó la UE como una organización internacional donde los Estados Miembros depositaron su soberanía y delegaron su poder a las instituciones supranacionales. Dentro de este paradigma, el establecimiento de agenda de la UE se centraba en la relación entre los Estados Miembros y las instituciones supranacionales, y las relaciones agente-principal en la delegación de poder y control del ejercicio de funciones ${ }^{4}$. En este marco institucional, los académicos estaban interesados en desenredar las preferencias y el comportamiento de los actores principales en el proceso de negociación y adopción de los actos legislativos de la UE, contra el trasfondo establecido por las reglas del juego y las oportunidades para ejercer influencia (ver especialmente el trabajo de Tsebelis y Garrett discutido abajo).

En los 90, una nueva idea cogió impulso. La UE ya no era vista como una entidad sui generis, sino más bien como un sistema político comparable con otros. Las herramientas de la política comparada fueron ganando popularidad entre los estudiosos de los actores y las instituciones europeas ${ }^{5}, \mathrm{y}$ las teorías y métodos de análisis de las políticas públicas también encontra-

${ }^{1}$ Peter Bachrach y Morton S. Baratz, «Two faces of power». The American Political Science Review 56, n.4 (1962): 947-952; Elmer Eric Schattschneider, The semi-sovereign people: A realist's view of democracy in America (New York: Holt, Rinehart and Winston, 1960).

2 Por ejemplo Roger W. Cobb y Charles D. Elder, «The politics of agenda-building: An alternative perspective for modern democratic theory». The Journal of Politics 33, n.4 (1971): 892-915; John W. Kingdon, Agendas, alternatives and public policies. (Boston: Little, Brown e Co., 1984).

${ }^{3}$ Por ejemplo Frank R. Baumgartner, Christoffer Green-Pedersen y Bryan D. Jones, ed. Comparative studies of policy agendas. Special Issue of the Journal of European Public Policy 13, n.7 (2006); Frank R. Baumgartner, Bryan D. Jones y John Wilkerson, ed. Comparative studies of policy dynamics. Special Issue of the Journal of Comparative Political Studies 44, n.8 (2011); Christoffer Green-Pedersen y Stefaan Walgrave, ed. Agenda setting, policies, and political systems: A comparative approach. Chicago: University of Chicago Press, 2014.

${ }^{4}$ Mark A. Pollack, «Delegation, agency, and agenda setting in the European Community». International Organization 51, n.1 (1997): 99-134; Mark A. Pollack, Delegation, agency and agenda setting in the treaty of Amsterdam. European Integration Online Papers, 3, n.6 (1999).

5 Simon Hix, «The study of the European Community: The challenge to comparative politics». West European Politics 17, n.1 (1994): 1-30 
ron su lugar en el estudio del proceso de adopción e implementación de las políticas de la UE ${ }^{6}$. Un artículo seminal de Guy Peters en 1994 allanó el terreno para una perspectiva completamente nueva en el establecimiento de agenda de la $\mathrm{UE}^{7}$. Ésta fue presentada como un proceso informal y abierto. Este resultado se consideraba que era resultado de dos factores interrelacionados: un gran número de actores, escenarios y puntos de acceso y un amplio abanico del alternativas políticas legítimas.

Desde entonces la investigación en este campo ha crecido significativamente, especialmente en la última década. Se han publicado una serie de estudios de casos centrados en un único asunto de políticas públicas. Los análisis también han señalado la dinámica de la formación de agenda en diferentes instituciones. El paradigma dominante ha cambiado desde la elección racional basada en los intereses hacia unas premisas de racionalidad limitada y más matizadas ${ }^{8}$. Además, se han hecho intentos para aplicar los modelos generales de configuración de agenda al caso de la UE, adaptándolos e identificando los elementos tras la naturaleza distintiva del escenario comunitario ${ }^{9}$. Más recientemente, se han hecho esfuerzos para desarrollar bases de datos cuantitativas sobre la distribución de la atención en la $\mathrm{UE}^{10}$. Estos esfuerzos sugieren que la investigación en la configuración de la agenda se ha movido cada vez más hacia la corriente principal de los estudios de configuración de agenda comparada, incluso si, como discutimos abajo, retiene algunos componentes específicos.

\section{II. Ámbito de investigación}

La investigación sobre el establecimiento de agenda en la UE se remonta a los años 90 y se asocia con dos marcos teóricos principales. El pri-

6 Jeremy Richardson, ed. Power and policy-making in the European Union. London: Routledge, 1996.

7 B. Guy Peters, «Agenda-setting in the European Community». Journal of European Public Policy, 1, n.1 (1994): 9-26.

${ }^{8}$ Aunque esta primera aproximación no ha sido rechazada, se aplica con menos frecuencia.

9 Sebastiaan Princen y Mark Rhinard, «Crashing and creeping: Agenda-setting dynamics in the European Union». Journal of European Public Policy 13, n.7 (2006): 1119-1132; Sebastiaan Princen, «Agenda-setting in the European Union: A theoretical exploration and agenda for research». Journal of European Public Policy 14, n.1 (2007): 21-38; Sebastiaan Princen, «Agenda-setting strategies in EU policy processes». Journal of European Public Policy 18, n.7 (2011): 927-943.

10 Petya Alexandrova et al. «Measuring the European Council agenda: Introducing a new approach and dataset». European Union Politics 15, n.1 (2014): 152-167. 
mero se fundamenta en premisas de la elección racional sobre la naturaleza y comportamiento humanos. Esta perspectiva toma una aproximación de negociación institucionalista y se centra en el poder de configuración de la agenda legislativa, es decir, en la capacidad de ciertos actores de hacer proposiciones que otros participantes del proceso de toma de decisiones no tengan capacidad de modificar. Su principal contribución se relaciona con la idea que las instituciones particulares de la UE pueden actuar como configuradores de agenda condicionales. El segundo marco teórico proviene de la literatura del análisis de políticas públicas y está basado en premisas de la racionalidad limitada. Dentro de ella, se pueden distinguir una serie de modelos teóricos, en particular las corrientes múltiples y el equilibrio interrumpido. Hay características distintivas a tener en cuenta en relación a la importancia de la presentación del problema y a las limitaciones en el comportamiento de los actores sobre distintos tipos de obstáculos cognitivos e institucionales a los que estos se pueden enfrentar.

\section{La aproximación teórica racional}

La aproximación de la elección racional al establecimiento de agenda asume que las instituciones de la UE y los Estados Miembro (representados en el Consejo de Ministros) tienen preferencias fijas que intentan optimizar durante la toma de decisiones de la UE. El poder de establecer la agenda es un instrumento que da ventaja en la formulación de decisiones. Esta aproximación teórica se hizo prominente al abordar las relaciones interinstitucionales y el balance de poder entre las instituciones en los distintos procedimientos de toma de decisiones de la UE.

En los primeros estudios el poder de configuración de agenda se veía como la «habilidad de hacer proposiciones que fueran difíciles de enmendar» ${ }^{11}$. Se analizó conjuntamente con (y hasta cierto punto en oposición a) el poder de veto, que representa la habilidad de rechazar proposiciones existentes. Los conceptos se aplicaron a las tres principales instituciones europeas - la Comisión Europea, el Consejo y el Parlamento Europeo (PE) - y se hizo una distinción entre los distintos procesos de toma de decisiones. La Comisión era considerada como el actor con un poder de configuración de agenda condicional en el proceso de consulta, mientras que este poder era compartido (o disputado) con el EP en el procedimiento de

11 George Tsebelis y Geoffrey Garrett, «Agenda setting power, power indices, and decision making in the European Union». International Review of Law and Economics 16, n.3 (1996): 345-361, p. 346. 
cooperación ${ }^{12}$. La validez de esta afirmación también ha sido cuestionada ${ }^{13}$. En el procedimiento de codecisión existía consenso sobre el poder de configuración de agenda del Consejo, pero el papel del PE se ponía en duda. Con la introducción de este procedimiento en el tratado de Maastricht el PE se convirtió para algunos autores en un configurador de agenda aún más débil ${ }^{14} \mathrm{o}$, para otros, más fuerte ${ }^{15}$. Este campo de investigación se fundamentaba en la elaboración de modelos espaciales formales de la negociación institucional, y se centraban básicamente en consideraciones teóricas. También se realizaron varios estudios empíricos, la mayoría sobre el $\mathrm{PE}^{16}$.

Más recientemente, las aproximaciones a la teoría de la elección racional en el establecimiento de agenda se han extendido al análisis de más actores en el sistema de la UE, como el Consejo de Gobierno del $\mathrm{BCE}^{17}$ o la Convención Europea responsable de la redacción del tratado constitucional de la $\mathrm{UE}^{18}$. Sin embargo, este tipo de investigaciones son algo excepcional, y el principal enfoque de análisis ha permanecido en las tres instituciones en el centro del proceso legislativo. Por otro lado, las preguntas de investigación planteadas han ido más allá de la medida y distinción del nivel de

12 Por ejemplo Bernard Steunenberg, «Decision making under different institutional arrangements: Legislation by the European Community». Journal of Institutional and Theoretical Economics/ Zeitschrift Für Die Gesamte Staatswissenschaft 150, n.4 (1994): 642-669; George Tsebelis, «The power of the European Parliament as a conditional agenda setter». The American Political Science Review 88, n.1 (1994): 128-142; Tsebelis e Garrett, «Agenda setting power, power indices...».

${ }_{13}$ Ver por ejemplo Peter Moser, «The European Parliament as a conditional agenda setter: What are the conditions? A critique of Tsebelis (1994)». The American Political Science Review 90, n.4 (1996): 834-838.

${ }_{14}$ Por ejemplo. Tsebelis y Garrett, «Agenda setting power, power indices...»; George Tsebelis e Geoffrey Garrett, «Agenda setting, vetoes and the European Union's co-decision procedure». The Journal of Legislative Studies 3, n.3 (1997): 74-92.

15 Por ejemplo Christophe Crombez, «The co-decision procedure in the European Union». Legislative Studies Quarterly 22, n.1 (1997): 97-119; Roger M. Scully, «The European Parliament and co-decision: A rejoinder to Tsebelis and Garrett». The Journal of Legislative Studies 3, n.3 (1997): 93-103.

16 Por ejemplo Thomas König y Mirja Pöter, «Examining the EU legislative process: The relative importance of agenda and veto power». European Union Politics 2, n.3 (2001): 329351; George Tsebelis y Anastassios Kalandrakis, «The European Parliament and environmental legislation: The case of chemicals». European Journal of Political Research 36, n. ${ }^{\circ} 1$ (1999): 119-154; George Tsebelis et al. «Legislative procedures in the European Union: An empirical analysis». British Journal of Political Science 31, n. ${ }^{\circ} 4$ (2001): 573-599.

17 Ansgar Belke y Barbara von Schnurbein, «European monetary policy and the ECB rotation model. Voting power of the core versus the periphery». Public Choice 151, n.1 (2012): 289-323.

18 George Tsebelis y Sven-Oliver Proksch, «The art of political manipulation in the European Convention». Journal of Common Market Studies 45, n. ${ }^{\circ} 1$ (2007): 157-186. 
poder de los actores, y se han centrado en los efectos del poder de configuración de agenda en los distintos aspectos del proceso legislativo ${ }^{19}$.

\section{La aproximación de la racionalidad limitada}

El segundo enfoque en el estudio del establecimiento de agenda de la UE considera que las premisas racionales de preferencias fijas así como la maximización de la utilidad de los objetivos por parte de los actores plantean algunos problemas. Basándose en introspecciones de la psicología conductual, se ha argumentado que los seres humanos y las instituciones políticas tienen una racionalidad limitada. Esto sería un resultado de la capacidad limitada de los responsables de adoptar decisiones a la hora de buscar y procesar información, así como por la ambigüedad e incertidumbre del entorno en el que operan ${ }^{20}$. Esta línea de investigación se centra en investigar porqué se presta prestar atención a determinados asuntos (y se descarta otros), y que capacidad etiene cada actor en cada asunto ${ }^{21}$. La orientación hacia los problemas es más típica de los constructivistas, ya que los problemas se consideran que son definidos por los actores que tienen capacidad para configurar la agenda ${ }^{22}$. Un problema se vuelve políticamente relevante no (únicamente) por su naturaleza intrínseca o severidad, sino (también) porque ciertos actores tienen más capacidad que otros para introducir ciertos asuntos en la agenda.

Un marco analítico relevante dentro de esta aproximación es el denominado modelo de corrientes múltiples de John Kingdon ${ }^{23}$. Dentro de este enfoque, existirían tres corrientes distintas: problemas, política y políticas públicas. Estos tres elementos se han de unir para que un asunto pueda ser introducido con éxito en la agenda de las instituciones políticas. El modelo de Kingdon fue una excepción de la hasta entonces visión dominante y ciertamente limitadora del proceso político como un ciclo en el que el establecimiento de agenda era una fase previa a la posterior formulación, decisión, implementación, y evaluación de políticas públicas. Ciertas soluciones (la

19 Ver por ejemplo. Anne Rasmussen y Dimiter Toshkov, «The inter-institutional division of power and time allocation in the European Parliament». West European Politics 34, n..$^{\circ} 1$ (2011): 71-96 distribución del tiempo en el PE .

${ }^{20}$ Bryan D. Jones «Bounded rationality». Annual Review of Political Science 2, n. 1 (1999): 297-321.

${ }^{21}$ Bachrach y Baratz, «Two faces of power»; Cobb e Elder, «The politics of agendabuilding».

22 David A. Rochefort y Roger W. Cobb, The politics of problem definition: Shaping the policy agenda (Lawrence, KS: University of Kansas Press, 1994).

23 Kingdon, Agendas, alternatives and public policies. 
corriente de las políticas públicas) pueden existir previamente a la configuración de la agenda, y estar esperando la oportunidad para ponerse encima de la mesa - algo que puede darse en el momento adecuado, que es cuando el asunto (la corriente del problema) se hace relevante entre los que diseñan las políticas y la opinión pública (la corriente de la política) - . Esto puede ocurrir cuando se abre una ventana de oportunidad - por ejemplo, como consecuencia de eventos extraordinarios - y el momento es aprovechado por actores y emprendedores políticos extremadamente hábiles.

Varios estudios han aplicado este modelo teórico a la UE, incorporando las características específicas del sistema político de la UE ${ }^{24}$. Otros se han centrado en aspectos determinados de la teoría. Por ejemplo, el papel de los emprendedores políticos se ha estudiado de un modo extensivo, con la Comisión desempeñando este papel. Sin embargo, la habilidad de otros actores institucionales o grupos externos también han sido analizadas (ver la sección siguiente). La capacidad para eliminar o «desconfigurar» ciertos temas en la agenda política también se han analizado empíricamente ${ }^{25}$.

La teoría del equilibrio interrumpido se ha desarrollado con el objeto de explicar las dinámicas y los mecanismos de cambio en las políticas públicas. Esta teoría sostiene que la mayoría de las veces, estos que formulan las políticas ignoran la información que existe sobre muchos de los problemas de las propias políticas. Es por esta razón, que las políticas son mayoritariamente estables o cambian de un modo muy incremental. Sin embargo, en algunas ocasiones, determinados temas alcanzan un umbral lo suficientemente importante que es prácticamente imposible ignorarlos; ello, puede ocasionar reacciones exagerados o repuntes de atención. Este modelo puede aplicarse de dos modos distintos - en estudio de casos de asuntos ${ }^{26}$ individuales, o en análisis longitudinales entre asuntos a largo plazo - ambos han encontrado su lugar en la comunidad académica de la $\mathrm{UE}^{27}$.

${ }^{24}$ Robert Ackrill y Adrian Kay,. «Multiple streams in EU policy-making: The case of the 2005 sugar reform». Journal of European Public Policy 18, n. 1 (2011): 72-89; Ian Bache, «Measuring quality of life for public policy: An idea whose time has come? Agenda-setting dynamics in the European Union». Journal of European Public Policy 20, n.1 (2013): 21-38; Paul Copeland e Scott James, «Policy windows, ambiguity and commission entrepreneurship: Explaining the relaunch of the European Union's economic reform agenda». Journal of European Public Policy 21, n.1 (2014): 1-19; Nikolaos Zahariadis, «Ambiguity and choice in European public policy». Journal of European Public Policy 15, n.4 (2008): 514-530.

${ }_{25}$ Petya Alexandrova, «Upsetting the agenda: The clout of external focusing events in the European Council», Journal of Public Policy 35, n. 3 (2015): 505-530; Princen e Rhinard, «Crashing and creeping...».

${ }^{26}$ Bryan D. Jones e F. R. Baumgartner. The politics of attention. How government prioritizes problems. Chicago: University of Chicago Press, 2005.

27 Sebastiaan Princen, «Punctuated equilibrium theory and the European Union». Journal of European Public Policy 20, n.6 (2013): 854-870. 
Los casos de estudio se centran generalmente en las nociones clave de los escenarios de la acción política, así como de las imágenes que transmite la política ${ }^{28}$. El concepto de escenarios de la acción política hace referencia a los escenarios formales de toma de decisiones en el sistema político ${ }^{29}$, y diferentes escenarios en ocasiones son más receptivos a ciertos problemas que a otros. Como consecuencia, los actores interesados en hacer escalar determinados asuntos en la agenda llevan a cabo lo que se conoce como «venue shopping»: enfrentados a múltiples puntos de acceso, abordan el escenario donde es más probable que sus peticiones reciban atención ${ }^{30}$. Una estrategia relacionada consiste en intentar redefinir la imagen de un asunto ligándolo a unos marcos que tienen una probabilidad más alta de éxito en la lucha por la atención política ${ }^{31}$. La interacción entre escenarios e imágenes es crucial para comprender el establecimiento de agenda ${ }^{32}$ y el cambio de las políticas en un sentido más amplio ${ }^{33}$. Un buen ejemplo de esto es la política migratoria de la UE. La Migración se convirtió en competencia de la UE cuando los gobiernos decidieron afrontar el control migratorio y los problemas que este generaba en algunos estados, en este contexto los países de la UE se percataron de que europeizar esta política era el escenario más adecuado para sus intereses. Lo lograron vinculando la migración con la seguridad, y presentando el escenario supranacional como una vía mejor para abordar ambos asuntos ${ }^{34}$.

${ }^{28}$ Frank R. Baumgartner y Bryan D. Jones. Agendas and instability in American politics (Chicago: University of Chicago Press, 2009, 2a ed.).

29 Sebastiaan Princen, Agenda-setting in the European Union. Basingstoke: Palgrave Macmillan, 2009, p. 11.

${ }_{30}$ Por ejemplo Borja García, «From regulation to governance and representation: Agenda-setting and the EU's involvement in sport». Entertainment and Sports Law Journal 5, n. ${ }^{\circ} 1$ (2007); Virginie Guiraudon, «European integration and migration policy: Vertical policy-making as venue shopping». JCMS: Journal of Common Market Studies 38, n. 2 (2000): 251-271; Christian Kaunert y Sarah Léonard, «The development of the EU asylum policy: Venue-shopping in perspective». Journal of European Public Policy 19, n. 9 (2012): 13961413.

${ }^{31}$ Por ejemplo Falk Daviter, Policy framing in the European Union (Basingstoke: Palgrave Macmillan, 2011); Annabelle Littoz-Monnet, «Agenda-setting dynamics at the EU level: The case of the EU cultural policy». Journal of European Integration 34, n. ${ }^{\circ} 5$ (2012): 505-522; Mark Rhinard, Framing Europe: The policy shaping strategies of the European Commission (Dordrecht: Martinus Nijnhoff Publishers, 2010).

32 Por ejemplo Paul J. Stephenson, «Image and venue as factors mediating latent spillover pressure for agenda-setting change». Journal of European Public Policy 19, n. 6 (2012): 796-816; Bryan Wendon, «The Commission as image-venue entrepreneur in EU social policy». Journal of European Public Policy 5, n. ${ }^{\circ} 2$ (1998): 339-353.

33 Sebastiaan Princen, «Venue shifts and policy change in EU fisheries policy». Marine Policy 34, n. ${ }^{\circ} 1$ (2010): 36-41.

${ }^{34}$ Guiraudon, «European integration and migration policy...». 
Mientras que el análisis de la atención a los temas y dinámicas de la agenda ha proliferado a través de los estudios de caso, todavía no existen muchas investigaciones de naturaleza sistemática sobre la distribución de la atención y los cambios en ésta. Sin embargo, algunos autores han avanzado en esta dirección y han examinado temas tan relevantes como el presupuesto de la $\mathrm{UE}^{35}$ y la agenda política del Consejo Europeo ${ }^{36}$.

Entre las teorías basadas en premisas de racionalidad limitada, el marco de las coaliciones promotoras ${ }^{37}$ en la UE ha tenido una relevancia especial. Dicho marco ha estudiado las estructuras de las actitudes y las creencias, vistas como herramientas poderosas para el cambio de las políticas europeas. En este sentido, se forman coaliciones entre distintos actores, que buscan influir en las decisiones en un área política determinada, y estas coaliciones se mantienen unidas por creencias e intereses compartidos. Las distintas coaliciones de actores compiten entre sí dentro de los subsistemas de las políticas. El cambio de las políticas (o su ausencia) es el resultado de la competición que se establece entre coaliciones alternativas, o del aprendizaje de políticas. El marco de las coaliciones promotoras considera el venue-shopping como una estrategia posible para maximizar las ventajas $^{38}$. Por tanto, los estudios de venue shopping en la UE también pueden asociarse a esta aproximación. Las investigaciones que aplican el enfoque completo de esta perspectiva analítica en la UE tienen limitaciones ${ }^{39}$. No obstante, la noción de las coaliciones promotoras es una heurística estable-

35 Frank R. Baumgartner, Martial Foucault y Abel François, «Public budgeting in the EU Commission». Politique Européenne 38, n. ${ }^{\circ} 3$ (2012): 124-153; Manuele Citi, «EU budgetary dynamics: Incremental or punctuated equilibrium?». Journal of European Public Policy 20, n. 8 (2013): 1157-1173.

${ }^{36}$ Petya Alexandrova, Marcello Carammia y Arco Timmermans, «Policy punctuations and issue diversity on the European Council agenda». Policy Studies Journal 40, n. 1 (2012): 69-88; Petya Alexandrova, Marcello Carammia y Arco Timmermans. «EU high politics: The policy agenda of the European Council, 1975-2010». En The European Council and European governance. The commanding heights of the EU, editado por F. Foret e Y. Rittelmeyer, 53-72. London: Routledge: 2014.

${ }^{37}$ Hank C. Jenkins-Smith, «Analytical debates and policy learning: Analysis and change in the federal bureaucracy». Policy Sciences 21, n. ${ }^{\circ} 2$ (1988): 169-211; Paul A. Sabatier, «An advocacy coalition framework of policy change and the role of policy-oriented learning therein». Policy Sciences 21, n. 2 (1988): 129-168.

${ }^{38}$ Paul A. Sabatier, «The advocacy coalition framework: Revisions and relevance for Europe». Journal of European Public Policy 5, n. ${ }^{\circ} 1$ (1998): 98-130.

${ }^{39}$ Karl Magnus Johansson, «Tracing the employment title in the Amsterdam Treaty: Uncovering transnational coalitions». Journal of European Public Policy 6, n. ${ }^{\circ} 1$ (1999): 85101; Sonia Mazey, «The European Union and women's rights: From the Europeanization of national agendas to the nationalization of a European agenda?». Journal of European Public Policy 5, n. ${ }^{\circ} 1$ (1998): 131-152; Jeremy Richardson, «EU water policy: Uncertain agendas, shifting networks and complex coalitions». Environmental Politics 3, n. ${ }^{\circ}$ (1994): 139-167. 
cida en el análisis de cooperación y la competición entre actores políticos en la UE.

\section{Enfoque empírico de la investigación en el establecimiento de la agenda de la UE}

Con respecto al enfoque empírico de la investigación del establecimiento de agenda de la UE, se pueden distinguir dos ámbitos principales. El primero gira en torno a los actores o escenarios. Aquí el principal sujeto son las instituciones de la UE y los Estados Miembro. Los análisis buscan aclarar los papeles en el establecimiento de la agenda y los poderes de los actores individuales en diversos escenarios o centrarse más en las interacciones y su significado para la agenda de la UE. El segundo ámbito empírico se centra en temas específicos y busca esclarecer los procesos. De este modo, el interés está en un asunto o área política concreta y su evolución temporal. Una pregunta de investigación relevante es esta relacionada con las razones tras el origen de los asuntos y su priorización a nivel europeo. Los estudios pueden incorporar ambas perspectivas pero el enfoque es normalmente en uno de ellos.

\section{Actores y escenarios}

Como consecuencia del derecho reconocido en los tratados a la Comisión Europea para generar iniciativas políticas, esta puede ser vista como un poderoso configurador de la agenda. No obstante, también puede estar limitada ya que necesita tener en cuenta una amplia gama de factores, como pueden ser las opiniones de los colegisladores, las peticiones informales del Consejo Europeo y la planificación de la presidencia de la UE ${ }^{40}$. Múltiples estudios han demostrado que la Comisión actúa como un emprendedor político hábil y sofisticado en determinados asuntos o dentro de determinados dominios políticos ${ }^{41}$. Incluso puede innovar institucionalmente para refor-

40 Ver, por ejemplo, Anne Rasmussen, «Challenging the commission's right of initiative? Conditions for institutional change and stability». West European Politics 30, n. ${ }^{\circ} 2$ (2007): 244-264.

${ }_{41}$ Jakob Edler y Andrew D. James, «Understanding the emergence of new science and technology policies: Policy entrepreneurship, agenda setting and the development of the European framework programme». Research Policy 44, n. ${ }^{\circ} 6$ (2015): 1252-1265; Neil Fligstein, «Institutional entrepreneurs and cultural frames - the case of the European Union's single market program». European Societies 3 n. $^{\circ} 3$ (2001): 261-287; Paulette Kurzer y Alice Cooper, «Hold the croissant! The European Union declares war on obesity». Journal of Euro- 
zar su propia influencia sobre el establecimiento de la agenda ${ }^{42}$. El éxito o no de sus esfuerzos depende de un número determinado de condiciones, algunas de las cuales también han sido analizadas ${ }^{43}$. Las dinámicas internas también son relevantes para el establecimiento de la agenda, tal y como han demostrado algunas investigaciones recientes ${ }^{44}$. En realidad, el papel de la Comisión en el establecimiento de agenda en las distintas áreas depende de sus direcciones generales. Sus estrategias de cooperación a la hora de enmarcar el problema pueden mejorarlas ${ }^{45}$, mientras que el conflicto entre ellas las empeora ${ }^{46}$.

El Consejo Europeo ha jugado un papel importante en el establecimiento de la agenda de un modo informal ${ }^{47}$. La investigación existente ha evaluado los tipos de temas que la institución plantea ${ }^{48}$, las dinámicas del cambio político en su agenda ${ }^{49}$, y los temas particulares en los que centra su atención ${ }^{50}$. Además, los estudios sugieren una distinción esencial entre esta institución y la Comisión a la hora de establecer la agenda ${ }^{51}$. En ocasiones, las dos instituciones interactúan y pueden contribuir conjuntamente (o en

pean Social Policy 21, n. 2 (2011): 107-119; Tomas Maltby, «European Union energy policy integration: A case of European Commission policy entrepreneurship and increasing supranationalism». Energy Policy 55, April (2013): 435-444; John Peterson, «Technology policy in Europe: Explaining the framework programme and eureka in theory and practice». JCMS: Journal of Common Market Studies 29, n. 3 (1991): 269-290; Paul J. Stephenson, «Talking space: The European Commission's changing frames in defining Galileo». Space Policy 28, n. ${ }^{\circ} 2$ (2012): 86-93

42 Jens Blom-Hansen, «The origins of the EU comitology system: A case of informal agenda-setting by the Commission». Journal of European Public Policy 15, n. ${ }^{\circ} 2$ (2008): 208-226.

${ }^{43}$ Por ejemplo Alexandra Hennessy, «The role of agenda setting in pension market integration». Journal of European Integration 33, n. 5 (2011): 577-597.

${ }^{44}$ Miriam Hartlapp, Julia Metz y Christian Rauh, «Linking agenda setting to coordination structures: Bureaucratic politics inside the European Commission». Journal of European Integration $35, \mathrm{n}^{\circ} 4$ (2013): 425-441

${ }^{45}$ Littoz-Monnet, «Agenda-setting dynamics at the EU level...».

46 Jean Grugel y Ingi Iusmen, «The European Commission as guardian angel: The challenges of agenda-setting for children's rights». Journal of European Public Policy 20, n. ${ }^{\circ} 1$ (2013): 77-94.

47 Jan Werts, The European Council (Amsterdam: North Holland Elsevier Science Publishers B.V., 1992); Jan Werts, The European Council. (London: John Harper Publishing, 2008).

48 Alexandrova, Carammia y Timmermans, «Policy punctuations and issue diversity...»; Alexandrova, Carammia e Timmermans, «EU high politics...»; Wolfgang Wessels, Das politische System der Europäischen Union (Wiesbaden: VS Verlag, 2008).

49 Alexandrova, Carammia y Timmermans, «Policy punctuations and issue diversity...».

50 Alexandrova, «Upsetting the agenda...»; Alexandrova, P. «Explaining political attention allocation with the help of issue character: Evidence from the European Council». European Political Science Review (de próxima publicación), doi:10.1017/S1755773915000107

${ }^{51}$ Princen y Rhinard, «Crashing and creeping». 
oposición al otro) a determinados resultados de configuración de agenda ${ }^{52}$. Finalmente, se ha discutido recientemente que las dinámicas de la formación de agenda en el Consejo Europeo sugieren que la institución está asumiendo progresivamente el papel del «gobierno de facto» de la UE ${ }^{53}$.

Las investigaciones empíricas del PE se han centrado principalmente en su papel en relación a las otras instituciones centrales de la $\mathrm{UE}^{54}$, aunque el impacto de los actores individuales dentro del PE, en particular los rapporteurs, también se ha analizado ${ }^{55}$. En la tradición de la elección racional, el $\mathrm{PE}$ ha aparecido con bastante frecuencia en análisis interinstitucionales conjuntos ${ }^{56}$.

En los últimos años, las investigaciones han ido más allá de las principales instituciones de la UE, y existe un creciente interés en el papel desempeñado por determinados individuos. Los actores individuales que han sido objeto de interés académico son los Altos Representantes de la Política Exterior Común y de Seguridad ${ }^{57}$, y su sucesor -el Alto Representante de Asuntos Exteriores y Política de Seguridad ${ }^{58}$ así como también el presidente de la Convención Europea ${ }^{59}-$.

52 Pierre Bocquillon y Mathias Dobbels, «An elephant on the 13th floor of the berlaymont? European Council and Commission relations in legislative agenda setting». Journal of European Public Policy 21, n. ${ }^{\circ} 1$ (2014): 20-38

53 Marcello Carammia, Sebastiaan Princen y Arco Timmermans, «From summitry to EU government. An agenda-formation perspective on the European Council». Journal of Common Market Studies (de próxima publicación), DOI: 10.1111/jcms.12346.

${ }^{54}$ Por ejemplo Simon Hix, «Constitutional agenda-setting through discretion in rule interpretation: Why the European Parliament won at Amsterdam». British Journal of Political Science 32, n. ${ }^{\circ} 2$ (2002): 259-280; König e Pöter, «Examining the EU legislative process...».

55 Ver Giacomo Benedetto, «Rapporteurs as legislative entrepreneurs: The dynamics of the codecision procedure in Europe's Parliament». Journal of European Public Policy 12, n. 1 (2005): 67-88; Hae-Won Jun,

${ }^{56}$ Geoffrey Garrett y George Tsebelis, «An institutional critique of intergovernmentalism». International Organization 50, n. ${ }^{\circ} 2$ (1996): 269-299; Sara Hagemann y Bjørn Høyland, «Bicameral politics in the European Union». JCMS: Journal of Common Market Studies 48, n. 4 (2010): 811-833; Pollack, «Delegation, agency, and agenda setting...»; Tsebelis, «The power of the European Parliament...»; George Tsebelis y Amie Kreppel, «The history of conditional agenda-setting in European institutions». European Journal of Political Research 33, n. 1 (1998): 41-71; Tsebelis et al. «Legislative procedures in the European Union».

57 Hylke Dijkstra, «Agenda-setting in the common security and defence policy: An institutionalist perspective». Cooperation and Conflict 47, n. ${ }^{\circ} 4$ (2012): 454-472.

58 Sophie Vanhoonacker y Karolina Pomorska, «The European External Action Service and agenda-setting in European foreign policy». Journal of European Public Policy 20, n. 9 (2013): 1316-1331.

59 Mareike Kleine, «Leadership in the European Convention». Journal of European Public Policy 14, n. 8 (2007): 1227-1248; Tsebelis e Proksch, «The art of political manipulation...». 
El papel desempeñado por los Estados Miembro en el establecimiento de la agenda también ha sido objeto de análisis a través de la presidencia rotatoria de la UE - un escenario con un claro potencial para generar impacto en el establecimiento de la agenda - . Las oportunidades para que las presidencias rotatorias puedan influir provienen del acceso privilegiado a la información de las preferencias del resto de estados y a las herramientas de control sobre los procedimientos de decisión, el establecimiento de las agendas de trabajo, la estructuración de las mismas y la exclusión de posibles temas de la agenda ${ }^{60}$. De acuerdo a un estudio empírico cuantitativo, la Presidencia parece no disponer de una ventaja sistémica en el control de la agenda del Consejo Europeo ${ }^{61}$. Sin embargo, múltiples estudios de caso han demostrado que la posición privilegiada de la presidencia permite a esta dirigir el proceso político instando a sus socios a prestar mayor atención a unos temas sobre otros. Ejemplos de esto son Dinamarca sobre la ampliación ${ }^{62}$, Suecia sobre la prevención de $\operatorname{conflictos}^{63}$ y las políticas antialcohol $^{64}$ y Polonia sobre la Asociación en la Europea Oriental ${ }^{65}$. Estos hallazgos muestran el potencial de investigación que tiene combinar visiones cualitativas con aproximaciones de naturaleza más cuantitativas y sistemáticas. Mientras que las primeras son más adecuadas para capturar los detalles y sutilezas de los asuntos políticos que se estudian, las últimas son necesarias para evitar generalizaciones equivocadas.

Más allá del enfoque sobre las instituciones de la UE y los Estados Miembro, existe un nicho de investigación que aborda la relación entre la UE y el nivel global en el establecimiento de agenda. Esta relación se analiza en ambas direcciones. Las organizaciones internacionales puede tener un impacto en la agenda de la UE, tal y como se ha mostrado en el marco de las reformas liberales del estado de bienestar y las políticas de mercado

60 Jonas Tallberg, «The agenda-shaping powers of the EU Council presidency». Journal of European Public Policy 10, n. 1 (2003): 1-19; Jonas Tallberg, «The power of the presidency: Brokerage, efficiency and distribution in EU negotiations». Journal of Common Market Studies 42, n. ${ }^{\circ} 5$ (2004): 999-1022.

61 Petya Alexandrova y Arco Timmermans, «National interest versus the common good: The presidency in European Council agenda setting». European Journal of Political Research 52, n. 3 (2013): 316-338.

${ }^{62}$ Lykke Friis, «The Danish presidency: "Wonderful Copenhagen”». Journal of Common Market Studies 41, Annual Review (2003): 49-51.

63 Annika Björkdahl, «Norm advocacy: A small state strategy to influence the EU». Journal of European Public Policy 15 n. 1 (2008), 135-154.

${ }^{64}$ Jenny Cisneros Örnberg, «Escaping deadlock - alcohol policy-making in the EU». Journal of European Public Policy 16, n. ${ }^{\circ} 5$ (2009): 755-773.

65 Bruno Vandecasteele, Fabienne Bossuyt y Jan Orbie. Unpacking the influence of the Council presidency on European Union external policies: The Polish council presidency and the eastern partnership. European Integration Online Papers (EIoP), 17, n. 5 (2013). 
de trabajo de la $\mathrm{OCDE}^{66}$ así como las políticas de la lucha contra obesidad de la OMS ${ }^{67}$. Pero, la UE también tiene capacidad para liderar la agenda global, tal y como se ha demostrado respecto a los esfuerzos de la Comisión en conseguir una convergencia global en la política de competición ${ }^{68}$.

\section{Procesos, asuntos y áreas de interés}

El segundo ámbito en el análisis empírico de la definición de agenda en la UE es bastante amplio y aborda los procesos, los asuntos y las áreas cubiertas. En este sentido, los ejemplos incluyen el estudio del mercado único ${ }^{69}$, la calidad de vida ${ }^{70}$, la ciencia y la tecnología ${ }^{71}$, las infraestructuras ferroviarias $^{72}$, las misiones civiles y militares de la $\mathrm{UE}^{73}$, el crimen organizado $^{74}$, el deporte ${ }^{75}$ y los fondos de cobertura,${ }^{76}$ entre otros. Aunque sea menos común, también se han llevado a cabo algunos análisis cruzados de temas y/o dominios políticos diferentes. Por ejemplo, Princen ha estudiado la evolución de las agendas políticas de salud y de pesca de la $\mathrm{UE}^{77}$ y un volumen editado trata aspectos diferentes de la agenda de política energética de la $\mathrm{UE}^{78}$.

Las políticas presupuestarias ocupan una posición central en los estudios empíricos. Dado que las políticas de gasto son transversales a una amplia gama de áreas políticas y son el resultado de un complejo proceso de negociación entre las instituciones de la UE (y otros actores), el presupuesto a veces es considerado como una representación de toda la agenda

66 Jörg Michael Dostal, «Campaigning on expertise: How the OECD framed EU welfare and labour market policies - and why success could trigger failure». Journal of European Public Policy 11, n. 3 (2004): 440-460.

67 Kurzer e Cooper (2011).

68 Chad Damro, «The new trade politics and EU competition policy: Shopping for convergence and co-operation». Journal of European Public Policy 13, n. ${ }^{\circ} 6$ (2006): 867-886.

${ }^{69}$ Fligstein, «Institutional entrepreneurs and cultural frames...».

${ }^{70}$ Bache, «Measuring quality of life for public policy...».

71 Edler y James, «Understanding the emergence of new science...».

${ }_{72}$ Stephenson, «Image and venue as factors mediating latent spillover pressure...».

73 Dijkstra, «Agenda-setting in the common security and defence policy...».

${ }^{74}$ Leticia Elias y Arco Timmermans, «Organised crime on the European Council agenda: Political attention dynamics». The European Review of Organised Crime 1, n. ${ }^{\circ} 1$ (2014): 160177.

75 García, «From regulation to governance and representation...».

76 Manuela Moschella, «Getting hedge funds regulation into the EU agenda: The constraints of agenda dynamics». Journal of European Integration 33, n.3 (2011): 251-266.

77 Princen, Agenda-setting in the European Union.

78 Jale Tosun, Sophie Biesenbender y Kai Schulze, ed. Energy policy making in the EU. Building the agenda (London: Springer, 2015). 
de la UE. En este sentido, estudiar el cambio en el contenido del presupuesto de la UE contribuye a entender el funcionamiento del sistema de la UE, incluyendo la forma en que se asigna la atención de las diversas políticas de gasto ${ }^{79}$. Sin embargo, el presupuesto también puede ser interpretado como un campo de batalla entre los actores institucionales. Cada actor lucha por aumentar la prominencia de los temas que son más relevantes para ellos mismos ${ }^{80}$.

\section{Conceptos clave y argumentos}

Un aspecto central del estudio de la agenda de la UE es la distinción entre los procesos formales e informales, como ha sugerido Pollack. Éste autor define el establecimiento formal de la agenda como el derecho (de la Comisión) o derecho condicional (del PE) para establecer la agenda formal o procedimental (del Consejo). El establecimiento informal de la agenda se refiere a la capacidad (en particular de la Comisión) de actuar como un emprendedor político, es decir, «su habilidad para definir los problemas y presentar propuestas que puedan generar consenso entre los que adoptan las decisiones finales» ${ }^{81}$. Estos dos modos $«$ se basan en la noción de agenda de toma de decisiones y agenda gubernamental» de Kingdon ${ }^{82}$. La primera de ellas se refiere al conjunto de las cuestiones que son objeto de la toma de decisiones, mientras que la segunda expresa el conjunto más amplio de temas que reciben atención en el sistema político.

En los procesos formales de toma de decisiones, el principal factor que condiciona el poder de fijar la agenda de los actores involucrados es el tipo de procedimiento legislativo ${ }^{83}$. Sin embargo, el prestigio y la aplicación de los diferentes procedimientos han cambiado a lo largo del tiempo, y lo más importante, el número de áreas políticas en las que la UE legisla se ha expandido enormemente. Las instituciones y los procesos informales son

${ }^{79}$ Baumgartner, Foucault y François, «Public budgeting in the EU Commission»; Citi, «EU budgetary dynamics...».

${ }^{80}$ Por Ejemplo Giacomo Benedetto y Bjorn Hoyland, «The EU annual budgetary procedure: The existing rules and proposed reforms of the convention and intergovernmental conference 2002-04». Journal of Common Market Studies 45, n.3 (2007): 565-587; Giacomo Benedetto, «The EU budget after Lisbon: Rigidity and reduced spending?». Journal of Public Policy 33, n.3 (2013): 345-369.

81 Pollack, «Delegation, agency, and agenda setting...», 121.

82 Kingdon, Agendas, alternatives and public policies.

83 Ver por ejemplo Tsebelis, «The power of the European Parliament...»; Tsebelis e Garrett, «Agenda setting power, power indices...»; Tsebelis e Garrett, «Agenda setting, vetoes and ...». 
clave para que un problema sea competencia de la UE. Los actores que tratan de poner un tema en la agenda de la UE necesitan, en primer lugar, afirmar la «Europeonidad» de ese tema. En otras palabras, es necesario enmarcarlo como un problema europeo que requiere soluciones europeas ${ }^{84}$. Esta estrategia es importante por varias razones. En primer lugar, el principio de subsidiariedad lo establece como requisito para motivar explícitamente la necesidad de acción de la UE. En segundo lugar, la movilización pública en la UE se ve limitada por la falta de responsabilidad directa de los que adoptan las decisiones y una esfera pública europea adecuada. La lógica informal del establecimiento de la agenda implica que los actores importantes van más allá de las instituciones centrales de la UE encargadas de la redacción y votación de la legislación de la UE.

Este elemento en el que diversos temas van escalando en la agenda es típico de sistemas políticos federales, en los que los escenarios políticos existen en múltiples niveles ${ }^{85}$. En la UE, esto es especialmente relevante debido a que el cambio de la jurisdicción política hacia el nivel de la UE es clave para el proceso de integración europea. En este sentido, el análisis de la definición de la agenda de la UE proporciona una nueva perspectiva para el estudio de la integración de la UE ${ }^{86}$.

También se ha desarrollado otra distinción con respecto a las diferentes vías a utilizar para que los problemas puedan llegar a la agenda de la UE. Princen y Rhinard diferencian entre una ruta de «alta política» que se origina en las reuniones del Consejo Europeo, y una ruta de «baja política», que comienza en los grupos de trabajo de expertos de la Comisión y del Consejo ${ }^{87}$. En la primera ruta, el establecimiento de la agenda se lleva a cabo por actores políticos de alto nivel. Como resultado, el proceso tiende a girar en torno a cuestiones relacionadas con riesgos políticos, y a veces se encauza a través de acontecimientos relevantes. Por el contrario, los problemas de baja ruta política se originan en redes tecnocráticas, por lo general en torno a la Comisión Europea, y son más bien impulsados por preocupaciones de naturaleza profesional. Aunque la política tecnocrática de la UE y la formulación de políticas tienen sus propios elementos distintivos, el establecimiento de la agenda por los especialistas es un posible patrón de formulación de políticas en todos los sistemas políticos, que de hecho se convierte en dominante cuando los problemas no están bajo la atención de los actores políticos de alto rango y/o bajo la opinión pública ${ }^{88}$.

\footnotetext{
${ }^{84}$ Princen, Agenda-setting in the European Union..

85 Baumgartner y Jones, Agendas and instability in American politics.

86 Princen, Agenda-setting in the European Union.

87 Princen y Rhinard, «Crashing and creeping...».

88 Baumgartner y Jones, Agendas and instability in American politics.
} 


\section{Herramientas de investigación}

Como hemos narrado hasta ahora, el campo de estudios sobre la configuración de la agenda en la UE es rico y diverso. Esto tiene implicaciones tanto positivas como negativas. Entre las negativas destaca la falta de un lenguaje común entre los investigadores que participan análisis. Este hecho dificulta la sistematicidad a la hora de evaluar hipótesis alternativas, lo que limita el progreso científico. Por otro lado, desde una perspectiva más positiva, la diversidad de perspectivas, enfoques, teorías y modelos empleados, contribuye a la creación de campo muy próspero de estudio.

Una forma de acercar los diferentes enfoques de investigación es el desarrollo de herramientas genéricas para el análisis. Los diversos investigadores pueden llevar a cabo, sobre este tipo de base, tanto investigaciones de naturaleza cuantitativa sobre una o varias instituciones como investigaciones cualitativas sobre cuestiones políticas específicas. Un ejemplo puede ser el enfoque de codificación de datos adoptado en el proyecto de las agendas políticas de la UE ${ }^{89}$. Este enfoque se nutre de un marco de naturaleza comparativa a nivel internacional - el Proyecto Comparado de Agendas ${ }^{90}$ - que reúne a más de treinta equipos de países diferentes ${ }^{91}$. Los datos recogidos permiten elaborar investigaciones de naturaleza comparada entre sistemas políticos diferentes en periodos largos de tiempo.

El Proyecto sobre Agendas Políticas de la UE ha presentado recientemente una base de datos ${ }^{92}$, clasificando la atención que ha dado el Consejo de la UE a distintos tipos de temas. Como los detalles de la base de datos están ya publicados ${ }^{93}$, aquí sólo presentamos un ejemplo para ilustrar cómo estos datos pueden encajar en diferentes investigaciones. A partir de esta base de datos sería posible llevar a cabo comparaciones entre instituciones.

Nos centramos en el ámbito de las políticas de inmigración y asilo, dado que en los últimos años han estado en el centro de atención diferentes problemas en esta área. Las fotos de barcos abarrotados en el Mediterráneo y las playas de Lesbos o Lampedusa se han convertido en el símbolo de un problema de escala mayor de los que la UE está acostumbrada a experimentar. La multiplicidad de actores afectados por el problema de maneras di-

89 «The project», European Union Policy Agendas Project, acceso el 22 de abril de 2016. www.policyagendas.eu

90 Comparative Agendas Project, acceso el 22 de abril de 2016, http://www.comparative agendas.net/

${ }^{91}$ La idea y la aproximación fueron inicialmente desarrolladlas por Frank Baumgartner y Bryan Jones con respecto los EUA para el proyecto, Policy Agendas Project (www.policy agendas.org)

${ }_{92}$ Se puede descargar en la página web del proyecto

93 Petya Alexandrova et al. «Measuring the European Council agenda...». 
recta o indirecta ha abonado un terreno para una variedad de perspectivas sobre cómo enmarcar el problema - desde los derechos humanos, a través de la criminalidad, hasta la carga económica o posibles oportunidades económicas que se pudieran generar, por mencionar algunos de los marcos utilizados más destacados - . La gravedad del problema y la imposibilidad de la UE para llegar a una posición común, en particular en el Consejo Europeo, se ha sumado a la urgencia y a la sensación de crisis.

La figura 1 ofrece una visión general de la atención a la inmigración en las reuniones del Consejo Europeo desde la primera cumbre regular de la institución en 1975 hasta finales de 2014. Esta figura muestra que, efectivamente, la atención a este ámbito ha sido mayor en el período más reciente (con la excepción de 1991). Pero incluso en los momentos en que la atención ha llegado a su punto más alto, este dominio ocupa menos del 9 por ciento del total de la agenda. Esto sugiere que puede haber límites al relieve de un tema si este está conectado a un problema en un campo político determinado. Por otra parte, la figura también ilustra la evolución en los flujos de atención (con aumentos y disminuciones), sobre todo desde el año 2009. La figura muestra que, por ejemplo, cada vez que la inmigración estaba en la agenda, la atención se centraba más en el asilo que en, por ejemplo, el control de fronteras. Esto es así hasta el año 2002, desde entonces la tendencia se ha invertido.

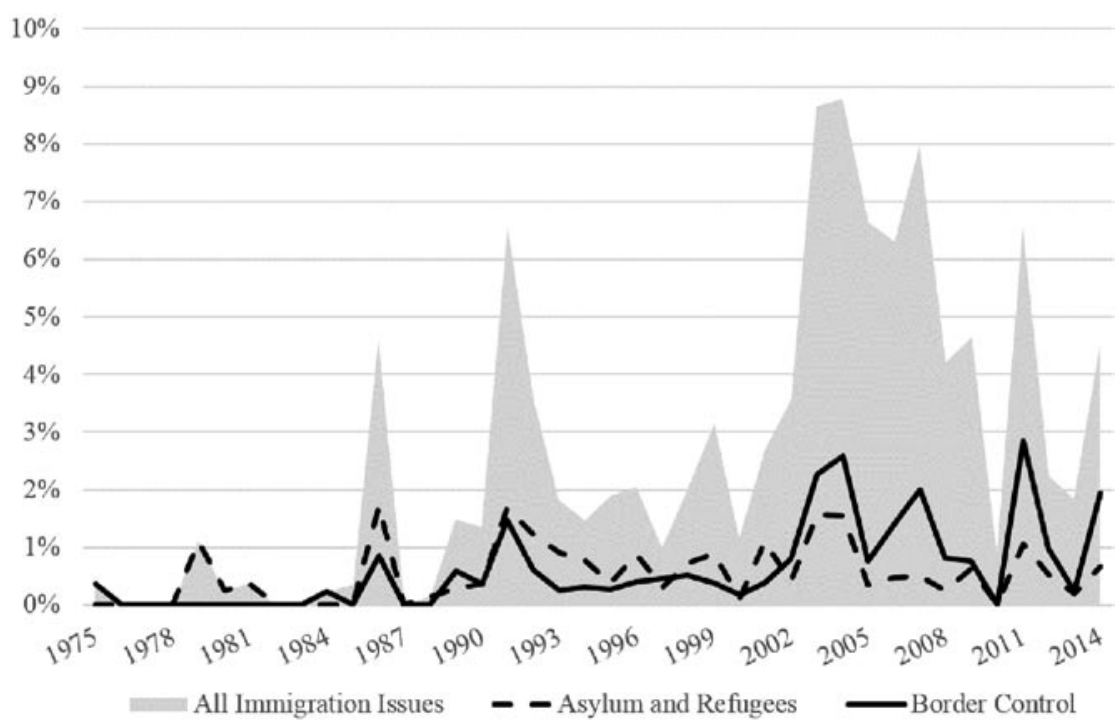

Figura 1

Atención a la política de inmigración y algunos temas dentro de la misma,

1975-2014 
La información que se presenta en la figura 1 puede servir de base para analizar las razones para asignar atención a la política de inmigración así como a posibles cambios en la misma, utilizando datos sobre el número de inmigrantes que viven en la UE o el número de solicitudes de asilo, las posiciones de los distintos Estados miembros (o actores dentro de estos, como los partidos políticos), así como las preferencias de los diferentes actores (por ejemplo, las encuestas de opinión pública, grupos de interés). Dado que los temas compiten entre sí por la atención de los políticos, las alianzas entre actores son posibles y, a veces se practican incluso con regularidad. Por ejemplo, el caso de inmigración (o temas particulares dentro de ella) puede estar relacionado con otros ámbitos políticos (o temas). Además, los datos pueden servir como punto de partida para abordar cuestiones más complejas, como por ejemplo, si la naturaleza de esta política afecta a las estrategias de fijación de la agenda.

\section{Conclusión}

El establecimiento de la agenda es relevante para el estudio de la UE en al menos dos sentidos ${ }^{94}$. En primer lugar, como hemos demostrado anteriormente, por sus múltiples contribuciones a la hora de entender el proceso general de elaboración de las políticas públicas de la UE. En segundo lugar, estudiar la inclusión de un tema en la agenda de la UE implica una mejor comprensión de la naturaleza y los resultados del proceso de integración. El estudio sobre el establecimiento de la agenda se utiliza cada vez más para conocer mejor el funcionamiento de un sistema político y sobre las preguntas políticas que este genera ${ }^{95}$. En ambos casos, todavía existen vías de investigación que quedan por explorar.

Una dirección relativamente inexplorada y, sin embargo, importante para el establecimiento de agenda de la UE es el estudio de las cuestiones que se mantienen fuera de la agenda, la llamada «no-decision» o el «segundo rostro» del poder ${ }^{96}$. Este tipo de estudios son muy limitados ${ }^{97}$ debido a la complejidad en los procesos de recopilar información de calidad. Sin embargo, sabiendo qué tipo de cuestiones no llegan al orden del día, y en qué condiciones esto es más probable que ocurra, podemos conocer mejor las implicaciones que esto tiene para comprender la configuración del establecimiento de agenda. Un enfoque que se centre únicamente en los pro-

\footnotetext{
94 Princen, Agenda-setting in the European Union.

${ }_{95}$ Green-Pedersen.y Walgrave, Agenda setting, policies, and political systems...

96 Bachrach y Baratz, «Two faces of power».

97 Cf. Princen, Agenda-setting in the European Union.
} 
blemas que llegan a estar en el orden del día es un análisis que, inevitablemente, está sesgado.

La recogida de datos a gran escala y el análisis sobre el interés de los temas en la UE es otra vía de investigación que necesita profundizar más. Los datos presentados en este artículo pueden ser un ejemplo. Enfocar las agendas de las diferentes instituciones en un sector político determinado puede ser otra ${ }^{98}$. Se debe de profundizar en ambas orientaciones. Esto ayudaría a desarrollar modelos más avanzados en la configuración de las agendas dentro del contexto de la UE que incorporan múltiples actores y niveles ${ }^{99}$, así como también poder verificar estos modelos con datos empíricos de calidad. Todo ello nos ayudaría a profundizar sobre la selección de los temas y los mecanismos de priorización de los principales actores de la UE, así como las posibles luchas de poder a la hora de fijar la agenda política.

Por otra parte, es preciso distinguir entre la fijación de la agenda y la toma de decisiones. Esto es importante con el fin de poder estudiar la relación entre estas dos etapas de la formulación de políticas públicas en la UE. Incluso fuera del contexto de la UE existe poca investigación sobre la relación entre el nivel de interés sobre un problema político y las políticas públicas adoptadas al respecto ${ }^{100}$. Es necesario obtener más información sobre las condiciones en las que el éxito en el establecimiento de agenda se traduce en éxito en la toma de decisiones. Esto implica una mayor atención a la direccionalidad del interés sobre un tema y a la contextualización de problemas en una perspectiva sistemática. Este tipo de investigación también nos ayudaría a conocer la diferencia que existe entre la capacidad en la respuesta retórica y en la respuesta eficaz de las políticas de la $\mathrm{UE}^{101}$, un área de investigación con una clara relevancia social que sigue estando en gran parte inexplorada ${ }^{102}$.

98 Princen, Agenda-setting in the European Union.

99 Princen, «Agenda-setting strategies in EU policy processes».

100 Bryan D. Jones, Heather, Larsen-Price y John Wilkerson, «Representation and American governing institutions». The Journal of Politics 71, n. ${ }^{\circ} 1$ (2009): 277.

${ }^{101}$ Sarah Binzer Hobolt y Robert Klemmensen, «Government responsiveness and political competition in comparative perspective». Comparative Political Studies 41, n. 3 (2008): 309-337.

102 Petya Alexandrova, Anne Rasmussen y Dimiter Toshkov, «Agenda responsiveness in the european council: Public priorities, policy problems and political attention». West European Politics, (de próxima publicación) doi:10.1080/01402382.2015.1104995 


\section{Bibliografía}

Ackrill, Robert, y Adrian Kay. «Multiple streams in EU policy-making: The case of the 2005 sugar reform». Journal of European Public Policy 18, n. 1 (2011): 72-89.

Alexandrova, Petya. «Upsetting the agenda: The clout of external focusing events in the European council», Journal of Public Policy 35, n. 3 (2015): 505-530.

Alexandrova, Petya. «Explaining political attention allocation with the help of issue character: Evidence from the European Council». European Political Science Review (de próxima publicación), doi:10.1017/S1755773915000107

Alexandrova, Petya, Marcello Carammia, Sebastiaan Princen y Arco Timmermans. «Measuring the European Council agenda: Introducing a new approach and dataset». European Union Politics 15, n.1 (2014): 152-167.

Alexandrova, Petya, Marcello Carammia y Arco Timmermans. «Policy punctuations and issue diversity on the European Council agenda». Policy Studies Journal 40, n.1 (2012): 69-88.

Alexandrova, Petya, Marcello Carammia y Arco Timmermans. «EU high politics: The policy agenda of the European Council, 1975-2010». En The European Council and European governance. The commanding heights of the EU, editado por F. Foret e Y. Rittelmeyer, 53-72. London: Routledge: 2014.

Alexandrova, Petya, Anne Rasmussen y Dimiter Toshkov. «Agenda responsiveness in the european council: Public priorities, policy problems and political attention». West European Politics (de próxima publicación), doi:10.1080/01402382. 2015.1104995

Alexandrova, Petya y Arco Timmermans, A. «National interest versus the common good: The presidency in European Council agenda setting». European Journal of Political Research 52, n.3 (2013): 316-338.

Bache, Ian. «Measuring quality of life for public policy: An idea whose time has come? Agenda-setting dynamics in the European Union». Journal of European Public Policy 20, n.1 (2013): 21-38.

Bachrach, Peter y Baratz, Morton S. «Two faces of power». The American Political Science Review 56, n.4 (1962): 947-952.

Baumgartner, Frank R., Martial Foucault y Abel François. «Public budgeting in the EU Commission». Politique Européenne 38, n. 3 (2012): 124-153.

Baumgartner, Frank R., Christoffer Green-Pedersen y Bryan D. Jones, ed. «Comparative studies of policy agendas». Special Issue of the Journal of European Public Policy 13, n.7 (2006): 959-1146.

Baumgartner, Frank R. y Bryan D. Jones. Agendas and instability in American politics. Chicago: University of Chicago Press, 2009 (2a ed.).

Baumgartner, Frank R., Bryan D. Jones y John Wilkerson, ed. «Comparative studies of policy dynamics». Special Issue of the Journal of Comparative Political Studies 44, n.8 (2011): 947-1119.

Belke, Ansgar y Barbara von Schnurbein. «European monetary policy and the ECB rotation model. Voting power of the core versus the periphery». Public Choice 151, n.1 (2012): 289-323. 
Benedetto, Giacomo. «Rapporteurs as legislative entrepreneurs: The dynamics of the codecision procedure in Europe's Parliament». Journal of European Public Policy 12, n.1 (2005): 67-88.

Benedetto, Giacomo. «The EU budget after lisbon: Rigidity and reduced spending?». Journal of Public Policy 33, n.3 (2013): 345-369.

Benedetto, Giacomo y Bjorn Hoyland. «The EU annual budgetary procedure: The existing rules and proposed reforms of the convention and intergovernmental conference 2002-04». Journal of Common Market Studies 45, n.3 (2007): 565-587.

Björkdahl, Annika. «Norm advocacy: A small state strategy to influence the EU». Journal of European Public Policy 15 n.1 (2008), 135-154.

Blom-Hansen, Jens. «The origins of the EU comitology system: A case of informal agenda-setting by the Commission». Journal of European Public Policy 15, n.2 (2008): 208-226.

Bocquillon, Pierre, y Mathias Dobbels. «An elephant on the 13th floor of the berlaymont? European Council and Commission relations in legislative agenda setting». Journal of European Public Policy 21, n.1 (2014): 20-38.

Carammia, Marcello, Sebastiaan Princen y Arco Timmermans. «From summitry to EU government. An agenda-formation perspective on the European Council». Journal of Common Market Studies (de próxima publicación), DOI: 10.1111/jcms.12346.

Cisneros Örnberg, Jenny. «Escaping deadlock - alcohol policy-making in the EU». Journal of European Public Policy 16, n.5 (2009): 755-773.

Citi, Manuele. «EU budgetary dynamics: Incremental or punctuated equilibrium?». Journal of European Public Policy 20, n.8 (2013): 1157-1173.

Cobb, Roger W. y Charles D. Elder. «The politics of agenda-building: An alternative perspective for modern democratic theory». The Journal of Politics 33, n.4 (1971): 892-915.

Comparatives Agendas Project. Acceso el 22 de abril de 2016. http://www. comparativeagendas.net/

Copeland, Paul y Scott James. «Policy windows, ambiguity and commission entrepreneurship: Explaining the relaunch of the European Union's economic reform agenda». Journal of European Public Policy 21, n.1 (2014): 1-19.

Crombez, Christophe. «The co-decision procedure in the European Union». Legislative Studies Quarterly 22, n.1 (1997): 97-119.

Damro, Chad. «The new trade politics and EU competition policy: Shopping for convergence and co-operation». Journal of European Public Policy 13, n.6 (2006): 867-886.

Daviter, Falk. Policy framing in the European Union. Basingstoke: Palgrave Macmillan, 2011.

Dijkstra, Hylke. «Agenda-setting in the common security and defence policy: An institutionalist perspective». Cooperation and Conflict 47, n.4 (2012): 454-472.

Dostal, Jörg Michael. «Campaigning on expertise: How the OECD framed EU welfare and labour market policies - and why success could trigger failure». Journal of European Public Policy 11, n.3 (2004): 440-460.

Edler, Jakob y Andrew D. James. «Understanding the emergence of new science and technology policies: Policy entrepreneurship, agenda setting and the de- 
velopment of the European framework programme». Research Policy 44, n.6 (2015): 1252-1265.

Elias, Leticia y Arco Timmermans. «Organised crime on the European Council agenda: Political attention dynamics». The European Review of Organised Crime 1, n.1 (2014): 160-177.

European Union Policy Agendas Project. «The project». Acceso el 22 de abril de 2016. http://www.policyagendas.eu/

Fligstein, Neil. «Institutional entrepreneurs and cultural frames - the case of the European Union's single market program». European Societies 3, n.3 (2001): 261287.

Friis, Lykke. «The Danish presidency: "Wonderful Copenhagen”». Journal of Common Market Studies 41, Annual Review (2003): 49-51.

García, Borja. «From regulation to governance and representation: Agenda-setting and the EU's involvement in sport». Entertainment and Sports Law Journal, 5, n.1 (2007): 1-13.

Garrett, Geoffrey y George Tsebelis. «An institutional critique of intergovernmentalism». International Organization 50, n.2 (1996): 269-299.

Green-Pedersen, Christoffer y Stefaan Walgrave, ed. Agenda setting, policies, and political systems: A comparative approach. Chicago: University of Chicago Press, 2014.

Grugel, Jean y Ingi Iusmen. «The European Commission as guardian angel: The challenges of agenda-setting for children's rights». Journal of European Public Policy 20, n.1 (2013): 77-94.

Guiraudon, Virginie. «European integration and migration policy: Vertical policymaking as venue shopping». JCMS: Journal of Common Market Studies 38, n.2 (2000): 251-271.

Hagemann, Sarah, y Bjørn Høyland. «Bicameral politics in the European Union». JCMS: Journal of Common Market Studies 48, n.4 (2010): 811-833.

Hartlapp, Miriam, Julia Metz y Christian Rauh. «Linking agenda setting to coordination structures: Bureaucratic politics inside the European Commission». Journal of European Integration 35, n.4 (2013): 425-441.

Hennessy, Alexandra. «The role of agenda setting in pension market integration». Journal of European Integration 33, n.5 (2011): 577-597.

Hix, Simon. «Constitutional agenda-setting through discretion in rule interpretation: Why the European Parliament won at Amsterdam». British Journal of Political Science 32, n.2 (2002): 259-280.

Hix, Simon. «The study of the European Community: The challenge to comparative politics». West European Politics 17, n.1 (1994): 1-30.

Hobolt, Sarah Binzer y Robert Klemmensen. «Government responsiveness and political competition in comparative perspective». Comparative Political Studies 41, n.3 (2008): 309-337.

Jenkins-Smith, Hank C. «Analytical debates and policy learning: Analysis and change in the federal bureaucracy». Policy Sciences 21, n.2 (1988): 169-211.

Johansson, Karl Magnus. «Tracing the employment title in the Amsterdam Treaty: Uncovering transnational coalitions». Journal of European Public Policy 6, n.1 (1999): 85-101. 
Jones, Bryan D. «Bounded rationality». Annual Review of Political Science 2, n.1 (1999): 297-321.

Jones, Bryan D. y Frank R. Baumgartner. The politics of attention. How government prioritizes problems. Chicago: University of Chicago Press, 2005.

Jones, Bryan D., Heather Larsen-Price y John Wilkerson. «Representation and American governing institutions». The Journal of Politics 71, n.1 (2009): $277-$ 290.

Jun, Hae-Won. «Supranational agenda setters in the European Union: Rapporteurs in the European Parliament». Korea Review of International Studies 16, n.1 (2013): 17-33.

Kaunert, Chriastian y Sarah Léonard. «The development of the EU asylum policy: Venue-shopping in perspective». Journal of European Public Policy 19, n.9 (2012): 1396-1413.

Kingdon, John W. Agendas, alternatives and public policies. Boston: Little, Brown e Co., 1984.

Kleine, Mareike. «Leadership in the European Convention». Journal of European Public Policy 14, n.8 (2007): 1227-1248.

König, Thomas y Mirja Pöter. «Examining the EU legislative process: The relative importance of agenda and veto power». European Union Politics 2, n.3 (2001): 329-351.

Kurzer, Paulette y Alice Cooper. «Hold the croissant! The European Union declares war on obesity». Journal of European Social Policy 21, n.2 (2011): 107-119.

Littoz-Monnet, Annabelle. «Agenda-setting dynamics at the EU level: The case of the EU cultural policy». Journal of European Integration 34, n.5 (2012): 505522.

Maltby, Tomas. «European Union energy policy integration: A case of European Commission policy entrepreneurship and increasing supranationalism». Energy Policy 55, April (2013): 435-444.

Mazey, Sonia. «The European Union and women's rights: From the Europeanization of national agendas to the nationalization of a European agenda?». Journal of European Public Policy 5, n.1 (1998): 131-152.

Moschella, Manuela. «Getting hedge funds regulation into the EU agenda: The constraints of agenda dynamics». Journal of European Integration 33, n.3 (2011): 251-266.

Moser, Peter. «The European Parliament as a conditional agenda setter: What are the conditions? A critique of Tsebelis (1994)». The American Political Science Review 90, n.4 (1996): 834-838.

Peters, B. Guy. «Agenda-setting in the European Community». Journal of European Public Policy, 1, n.1 (1994): 9-26.

Peterson, John. «Technology policy in Europe: Explaining the framework programme and eureka in theory and practice». JCMS: Journal of Common Market Studies 29, n.3 (1991): 269-290.

Pollack, Mark A. «Delegation, agency, and agenda setting in the European Community». International Organization 51, n.1 (1997): 99-134.

Pollack, Mark. «A Delegation, agency and agenda setting in the treaty of Amsterdam». European Integration Online Papers, 3, n.6 (1999): 1-19. 
Princen, Sebastiaan. «Agenda-setting in the European Union: A theoretical exploration and agenda for research». Journal of European Public Policy 14, n.1 (2007): 21-38.

Princen, Sebastiaan. Agenda-setting in the European Union. Basingstoke: Palgrave Macmillan, 2009.

Princen, Sebastiaan. «Venue shifts and policy change in EU fisheries policy». Marine Policy 34, n.1 (2010): 36-41.

Princen, Sebastiaan. «Agenda-setting strategies in EU policy processes». Journal of European Public Policy 18, n.7 (2011): 927-943.

Princen, Sebastiaan. «Punctuated equilibrium theory and the European Union». Journal of European Public Policy 20, n.6 (2013): 854-870.

Princen, Sebastiaan y Mark Rhinard. «Crashing and creeping: Agenda-setting dynamics in the European Union». Journal of European Public Policy 13, n.7 (2006): 1119-1132.

Rasmussen, Anne. «Challenging the commission's right of initiative? Conditions for institutional change and stability». West European Politics 30, n.2 (2007): 244-264.

Rasmussen, Anne y Dimiter Toshkov. «The inter-institutional division of power and time allocation in the European Parliament». West European Politics 34, n.1 (2011): 71-96.

Rhinard, Mark. Framing Europe: The policy shaping strategies of the European Commission. Dordrecht: Martinus Nijnhoff Publishers, 2010.

Richardson, Jeremy. «EU water policy: Uncertain agendas, shifting networks and complex coalitions». Environmental Politics 3, n.4 (1994): 139-167.

Richardson, Jeremy, ed. Power and policy-making in the European Union. London: Routledge, 1996.

Rochefort, David A. y Roger W. Cobb The politics of problem definition: Shaping the policy agenda. Lawrence, KS: University of Kansas Press, 1994.

Sabatier, Paul A. "An advocacy coalition framework of policy change and the role of policy-oriented learning therein». Policy Sciences 21, n.2 (1988): 129-168.

Sabatier, Paul A. «The advocacy coalition framework: Revisions and relevance for Europe». Journal of European Public Policy 5, n.1 (1998): 98-130.

Schattschneider, Elmer Eric. The semi-sovereign people: A realist's view of democracy in America. New York: Holt, Rinehart and Winston, 1960.

Scully, Roger M. «The European Parliament and co-decision: A rejoinder to Tsebelis and Garrett». The Journal of Legislative Studies 3, n.3 (1997): 93-103.

Stephenson, Paul J. «Image and venue as factors mediating latent spillover pressure for agenda-setting change». Journal of European Public Policy 19, n.6 (2012a): 796-816.

Stephenson, Paul J. «Talking space: The European Commission's changing frames in defining Galileo». Space Policy 28, n.2 (2012b): 86-93.

Steunenberg, Bernard. «Decision making under different institutional arrangements: Legislation by the European Community». Journal of Institutional and Theoretical Economics/ Zeitschrift Für Die Gesamte Staatswissenschaft 150, n.4 (1994): 642-669. 
Tallberg, Jonas. «The agenda-shaping powers of the EU Council presidency». Journal of European Public Policy 10, n.1 (2003): 1-19.

Tallberg, Jonas. «The power of the presidency: Brokerage, efficiency and distribution in EU negotiations». Journal of Common Market Studies 42, n.5 (2004): 999-1022.

Tosun, Jale, Sophie Biesenbender y Kai Schulze, ed. Energy policy making in the EU. Building the agenda. London: Springer, 2015.

Tsebelis, George. «The power of the European Parliament as a conditional agenda setter». The American Political Science Review 88, n.1 (1994): 128-142.

Tsebelis, George y Geoffrey Garrett. «Agenda setting power, power indices, and decision making in the European Union». International Review of Law and Economics 16, n.3 (1996): 345-361.

Tsebelis, George y Geoffrey Garrett. «Agenda setting, vetoes and the European Union's co-decision procedure». The Journal of Legislative Studies 3, n.3 (1997): 74-92.

Tsebelis, George, Christian B. Jensen, Anastassios Kalandrakis y Amie Kreppel. «Legislative procedures in the European Union: An empirical analysis». British Journal of Political Science 31, n.04 (2001): 573-599.

Tsebelis, George y Anastassios Kalandrakis. «The European Parliament and environmental legislation: The case of chemicals». European Journal of Political Research 36, n.1 (1999): 119-154.

Tsebelis, George y Amie Kreppel, A. «The history of conditional agenda-setting in European institutions». European Journal of Political Research 33, n.1 (1998): 41-71.

Tsebelis, George y Sven-Oliver Proksch. «The art of political manipulation in the European Convention». Journal of Common Market Studies 45, n.1 (2007): 157-186.

Vandecasteele, Bruno, Fabienne Bossuyt y Jan Orbie. Unpacking the influence of the Council presidency on European Union external policies: The Polish council presidency and the eastern partnership. European Integration Online Papers (EIoP), 17, n.5 (2013): 1-28.

Vanhoonacker, Sophie y Karolina Pomorska. «The European External Action Service and agenda-setting in European foreign policy». Journal of European Public Policy 20, n.9 (2013): 1316-1331.

Wendon, Bryan. «The Commission as image-venue entrepreneur in EU social policy». Journal of European Public Policy 5, n.2 (1998): 339-353.

Werts, Jan. The European Council. Amsterdam: North Holland Elsevier Science Publishers B.V., 1992.

Werts, Jan. The European Council. London: John Harper Publishing, 2008.

Wessels, Wolfgang. Das politische System der Europäischen Union. Wiesbaden: VS Verlag, 2008.

Zahariadis, Nikolaos. «Ambiguity and choice in European public policy». Journal of European Public Policy 15, n.4 (2008): 514-530. 


\section{Derechos de autor (Copyright)}

Los derechos de autor (distribución, comunicación pública, reproducción e inclusión en bases de datos de indexación y repositorios institucionales) de esta publicación pertenecen a la editorial Universidad de Deusto. El acceso al contenido digital de cualquier número de Cuadernos Europeos de Deusto (CED) es gratuito, transcurridos 6 meses desde su publicación. Los trabajos podrán descargarse, copiar y difundir, sin fines comerciales y según lo previsto por la ley. Así mismo, los trabajos editados en CED pueden ser publicados con posterioridad en otros medios o revistas, siempre que el autor indique con claridad y en la primera nota a pie de página que el trabajo se publicó por primera vez en CED, con indicación del número, año, páginas y DOI (si procede). 